\title{
Intensity correlation functions of dye lasers: Comparison of colored-gain-noise and colored-loss-noise models
}

\author{
J. M. Noriega \\ Departamento de Matemáticas, Facultad de Ciencias, Universidad de Oviedo, C/Calvo Sotelo, \\ $s / n, E-33007$ Oviedo, Spain
}

L. Pesquera and M. A. Rodríguez

Departamento de Física Moderna, Facultad de Ciencias, Universidad de Cantabria, Avenida de los Castros, s/n, E-39005 Santander, Spain

J. Casademunt

Department of Physics, University of Pittsburgh, Pittsburgh, Pennsylvania 15260

\author{
A. Hernández-Machado \\ Departamento de Estructura y Constituyentes de la Materia, Universidad de Barcelona, Avenida Diagonal, \\ 647, E-08028 Barcelona, Spain
}

(Received 21 December 1990; revised manuscript received 6 March 1991)

\begin{abstract}
The intensity correlation functions $C(t)$ for the colored-gain-noise model of dye lasers are analyzed and compared with those for the loss-noise model. For correlation times $\tau$ larger than the deterministic relaxation time $t_{d}$, we show with the use of the adiabatic approximation that $C(t)$ values coincide for both models. For small correlation times we use a method that provides explicit expressions of nonMarkovian correlation functions, approximating simultaneously short- and long-time behaviors. Comparison with numerical simulations shows excellent results simultaneously for short- and long-time regimes. It is found that, when the correlation time of the noise increases, differences between the gainand loss-noise models tend to disappear. The decay of $C(t)$ for both models can be described by a time scale that approaches the deterministic relaxation time. However, in contrast with the loss-noise model, a secondary time scale remains for large times for the gain-noise model, which could allow one to distinguish between both models.
\end{abstract}

\section{INTRODUCTION}

Statistical properties of dye lasers have been widely studied due to their anomalous character, as shown by the experimental results of Ref. [1]. Later experiments [2] suggested that these anomalies are due to fluctuations in the pump parameter. The standard theoretical model of dye-laser fluctuations can be formally obtained by replacing the loss parameter by a fluctuating quantity, and we will refer to it as the loss-noise model. A variety of calculations and simulations exist for this model in the literature, either in the white-noise limit [3] for the fluctuating loss parameter or considering a finite correlation time [4,5]. Experimental [6] and theoretical [7] work on dye lasers have been recently reviewed, and it seems that this model describes the experimental results and, in particular, intensity correlation functions $[8,9]$, if a finite correlation time is considered.

However, there exists [8] experimental evidence that identifies the pump laser as the source of noise. The natural way to account for these fluctuations is by introducing a fluctuating gain parameter model [10-15]. It has been shown [10] that in the white-noise limit the statistical properties predicted by loss- and gain-noise models are very different. Most of the qualitative predictions as- sociated with colored noise in the loss-noise model can be recovered within a white-gain-noise model [10]. However, it seems that the decay of $C(t)$ does not show two separated time scales [10-12], as observed in the experiments [9]. Therefore, a colored-noise model is required to describe the behavior of the intensity correlation function. In fact, this behavior can be obtained with both colored-loss-noise [9] and gain-noise [11,15] models.

Numerical simulations have shown [15] that differences between both models are masked by the correlation time of the noise $\tau$. The aim of this paper is to analyze the behavior of the intensity correlation function for the colored-gain-noise model and to compare it with that corresponding to the loss-noise model. We will consider situations above threshold. The case of $\tau$ much larger than the deterministic relaxation time $t_{d}$ is treated with the Stratonovich adiabatic approximation [16]. Due to the fact that $t_{d}$ decreases with the intensity, this case will occur far above threshold. When $\tau$ is smaller than $t_{d}$ we use a method [17] that provides explicit expressions of non-Markovian correlation functions to first order in $\tau$. This method contains two main ingredients, one dealing with the non-Markovian nature and the other with the nonlinearity of the process. The first one establishes the connection between the non-Markovian correlation func- 
tion, $C(t)$, and effective Markovian correlation functions. The second step is the application of the doubleexpansion method $[18,19]$ for the calculation of these functions. This procedure contains information of both short- and long-time regimes. In this way, we get an expression for $C(t)$ valid for the complete-time regime, in contrast with earlier results that were only useful in the short-time regime [20] or for the calculation of relaxation times [21]. We show that the method for non-Markovian correlation functions reproduces with great accuracy the simulation results.

Once we can reproduce the correlation functions in the complete regime, we make a detailed analysis of the correlation functions of the gain- and loss-noise models. Both the colored gain- [11,15] and loss-noise [9] models describe correctly the experimental results [6], in particular intensity correlation functions $[8,9]$. Then, it seems natural that they get closer when $\tau$ increases, as simulations indicate [15]. We show that when $\tau \gg t_{d}$, the normalized correlation functions for both models reproduce that of the noise. When $\tau<t_{d}$, we find that, as $\tau$ increases, the decay of $C(t)$ for times larger than $\tau$ can be described in both models by a time scale that approaches the deterministic time. However, a difference remains between both models due to a larger time scale with a decreasing amplitude with $\tau$ that is involved in the decay of $C(t)$ for the gain-noise model.

The paper is organized as follows. In Sec. II we introduce the gain-noise model, and an approximate expression for the stationary probability density is given. A method that provides an explicit expression for nonMarkovian correlation functions to first order in $\tau$ is presented in Sec. III, and a comparison with numerical simulations is also made. In Sec. IV we analyze the decay of the intensity correlation function for different values of $\tau$.

\section{GAIN-NOISE MODEL}

The gain-noise model for a single-mode dye laser on resonance is defined by the following stochastic equation for the intensity $[10,15]$ :

$$
\begin{gathered}
\partial_{t} I=I\left[-\alpha_{2}+\alpha_{1} /\left(1+I / \alpha_{1}\right)\right] \\
+\left[I /\left(1+I / \alpha_{1}\right)\right] p(t),
\end{gathered}
$$

where we have neglected the spontaneous emission noise because we consider situations above threshold, where it has a very small effect $[6,7]$. Here $\alpha_{1}$ and $\alpha_{2}$ are the gain and the loss parameters, respectively. $p(t)$ models fluctuations of the gain parameter, and, as usual, it is taken to be a Gaussian noise of zero mean and correlation:

$$
\left\langle p(t) p\left(t^{\prime}\right)\right\rangle=\frac{1}{\tau} \exp \left(-\frac{\left|t-t^{\prime}\right|}{\tau}\right),
$$

where $\tau$ is the correlation time of the noise.

If the nonlinear terms containing $\left(1+I / \alpha_{1}\right)^{-1}$ are expanded to first order in $I$, and the fluctuating saturation term $I^{2} p(t)$ is neglected, we obtain an equation of the form

$$
\partial_{t} I=I(\alpha-I)+I p(t),
$$

where $\alpha=\alpha_{1}-\alpha_{2}$. From a phenomenological point of view, the fluctuating term $I p(t)$ can be understood as arising from fluctuations of the loss parameter $\kappa$. So, we will refer to (2.3) as a loss-noise model.

The gain-noise model has, apart from the correlation time $\tau$, two independent parameters $\alpha_{1}$, and $\alpha_{2}$. The loss-noise model has only one independent parameter, $\alpha=\alpha_{1}-\alpha_{2}$, that corresponds to the pump parameter rescaled with respect to the noise intensity. The loss-noise model is obtained taking the limit $\alpha_{1}, \alpha_{2} \rightarrow \infty$ with $\alpha=\alpha_{1}-\alpha_{2}$ fixed. In the following we analyze the decay of $C(t)$ for different values of the parameter $\alpha / \alpha_{1}$. This parameter gives information on the importance of saturation effects in the fluctuations. When it is close to zero, these saturation effects are negligible and we recover the loss-noise model.

First, we give an approximate expression for the steady-state probability density $P_{\mathrm{st}}(I)$ associated with (2.1) and (2.2). The Fokker-Planck equation for the single probability density $P(I, t)$ is given for small correlation time $\tau$ by [22]

$$
\begin{aligned}
\frac{\partial}{\partial t} P(I, t) & =L(\tau) P(I, t) \\
& =-\frac{\partial}{\partial I} v(I) P(I, t)+\frac{\partial}{\partial I} g(I) \frac{\partial}{\partial I} h(I) P(I, t),
\end{aligned}
$$

with an effective Markovian Fokker-Planck operator $L(\tau)$, where

$$
h(I)=g(I)-\tau\left[v(I) g^{\prime}(I)-v^{\prime}(I) g(I)\right],
$$

$v(I)=I\left[-\alpha_{2}+\alpha_{1} /\left(1+I / \alpha_{1}\right)\right]$ is the deterministic term, and $g(I)=I /\left(1+I / \alpha_{1}\right)$ is the term multiplying the noise in Eq. (2.1).

The stationary solution of the Fokker-Planck equation (2.4) and (2.5) is defined in the interval in which the diffusion coefficient

$$
D(I)=\left(\frac{\alpha_{1} I}{\alpha_{1}+I}\right)^{2}\left(1-\frac{\tau \alpha_{2} I}{\alpha_{1}+I}\right)
$$

is positive. When $\tau \alpha_{2}>1$, the approximation introduces a spurious boundary at $I_{1}=\alpha_{1} /\left(\tau \alpha_{2}-1\right.$ ) (at $I_{1}=\tau^{-1}$ for any value of $\tau$ in the loss-noise model). For sufficiently small $\tau, I_{1}$ becomes very large, and there are not important effects due to the boundaries, but it can be interesting to consider larger values of $\tau$. There exists a procedure to explore larger values of $\tau$ avoiding the anomalous boundaries, consisting, essentially, in an exponentiation of an expansion in $\tau$ of $P_{\text {st }}$ [22]. However, this approximation gives good results only for negative pump parameters [23], that is, below threshold. Another type of approximation has been recently reported [24] that gives remarkably good results for the loss-noise model above threshold [23]. It can be even extended successfully to the case where spontaneous emission is included [23]. The basic idea is to extrapolate the diffusion coefficient obtained to first order in $\tau$ to larger values of 
$\tau$, in the following sense:

$$
\begin{aligned}
D(I) & =\left[\frac{\alpha_{1} I}{\alpha_{1}+I}\right]^{2}\left(1-\frac{\tau \alpha_{2} I}{\alpha_{1}+I}\right) \\
& \approx\left[\frac{\alpha_{1} I}{\alpha_{1}+I}\right]^{2}\left[1+\frac{\tau \alpha_{2} I}{\alpha_{1}+I}\right]^{-1} \\
& =\frac{\alpha_{1}^{2} I^{2}}{\left(\alpha_{1}+I\right)\left[\alpha_{1}+\left(1+\tau \alpha_{2}\right) I\right]} .
\end{aligned}
$$

For the loss-noise model this reduces to

$$
D(I)=I^{2}(1-\tau I) \approx \frac{I^{2}}{1+\tau I} .
$$

In this case, $D(I)$ given by (2.7) and (2.8) is always positive, avoiding the appearance of spurious boundaries. In the general case, this approximation consists of replacing $h(I)$ of Eq. (2.5) by $h_{p}(I)=g(I) /\{1-\tau g(I)[v(I)\}$ $\left.g(I)]^{-1}\right\}$. This reproduces the approximation of Ref. [25]. In this way we get an effective Markovian process, well defined for any value of the intensity. The stationary solution of the resulting Fokker-Planck equation is given for the gain-noise model by

$$
\begin{aligned}
& P_{\mathrm{st}}(I)=N_{1} I^{\alpha-1}\left[1+\left(1+\tau \alpha_{2}\right) I / \alpha_{1}\right] \\
& \times \exp \left[\left[\left(2+\tau \alpha_{2}\right) \frac{\alpha}{\alpha_{1}}-1\right] I\right. \\
&\left.-\frac{\left(1-\alpha / \alpha_{1}\right)}{2 \alpha_{1}}\left(1+\tau \alpha_{2}\right) I^{2}\right]
\end{aligned}
$$

and for the loss-noise model by

$P_{\mathrm{st}}(I)=N_{2}(1+\tau I) I^{\alpha-1} \exp \left[(\alpha \tau-1) I-\frac{\tau I^{2}}{2}\right]$,

where $N_{1}$ and $N_{2}$ are normalization constants.

It has been shown that the approximation given by (2.10) for the loss-noise model reproduces simulation results when the spontaneous emission noise can be neglected [23]. This corresponds to situations above threshold. Regarding the gain-noise model, we have compared the values of the mean intensity $\langle I\rangle$ and the normalized fluctuations $\left\langle(\delta I)^{2}\right\rangle /\langle I\rangle^{2}$ calculated with (2.9) with numerical simulations, obtaining a good agreement. Our results indicate that the mean intensity is nearly independent of the value of $\tau$. In fact, $\langle I\rangle$ is the same in the white-noise limit as in the limit $\tau \rightarrow \infty$ (see Sec. IV), and it coincides with the deterministic result. This expression, given by

$$
\langle I\rangle=\frac{\alpha}{1-\alpha / \alpha_{1}},
$$

can be then used as a good estimation of the mean intensity. We have also obtained that fluctuations become smaller with increasing $\tau$.

Before ending this section it is convenient to consider the linearization of Eq. (2.1) around the deterministic steady state $I_{0}=\alpha /\left(1-\alpha / \alpha_{1}\right)$. We use this approximation to compare with numerical simulations in the next section, and to interpret the results of the last section.
Then, we approximate the solution of (2.4) by $I=I_{0}+\delta I$, so that the linearized Langevin equation for $\delta I$ becomes

$$
\partial_{t}(\delta I)=-t_{d}^{-1} \delta I+\alpha p(t),
$$

where

$$
t_{d}=\left[\alpha\left(1-\alpha / \alpha_{1}\right)\right]^{-1} \approx\left[\langle I\rangle\left(1-\alpha / \alpha_{1}\right)^{2}\right]^{-1},
$$

which we call deterministic time. In this linear approximation the correlation function $\lambda(t)$ defined by

$$
\lambda(t)=\frac{\langle I(t) I(0)\rangle-\langle I\rangle^{2}}{\langle I\rangle^{2}}
$$

becomes

$$
\begin{aligned}
\lambda(t)= & \frac{\left(1-\alpha / \alpha_{1}\right)^{2}}{\left(1+\tau / t_{d}\right)\left(1-\tau / t_{d}\right)} \\
& \times\left[t_{d} \exp \left[-\frac{t}{t_{d}}\right)-\tau \exp \left[-\frac{t}{\tau}\right)\right] .
\end{aligned}
$$

The validity of this linear approximation has been analyzed in Ref. [15]. Generally speaking, the linearization is valid when fluctuations are small, that is,

$$
\begin{aligned}
\lambda(0) & =\frac{t_{d}^{2}\left(1-\alpha / \alpha_{1}\right)^{2}}{t_{d}+\tau} \\
& \approx \frac{1}{\langle I\rangle\left[1+\tau\langle I\rangle\left(1-\alpha / \alpha_{1}\right)^{2}\right]}<<.
\end{aligned}
$$

The range of validity then becomes wider with increasing $\tau$ and $\langle I\rangle$.

\section{ANALYTICAL CALCULATION OF NON-MARKOVIAN CORRELATION FUNCTIONS AND APPLICATION TO THE GAIN-NOISE MODEL}

\section{A. Method}

In this paper we will apply an analytical method for the calculation of steady-state correlation functions for general processes driven by colored noise with small correlation time. The method has been discussed in detail in Ref. [17], and provides systematically explicit expressions of correlation functions approximating simultaneously short- and long-time regimes. The nonMarkovicity is reduced to an effective Markovian formulation. This allows the systematic treatment of the nonlinearities by means of double expansions in high and low frequencies, as a generalization of the method introduced in Refs. [18 and 19] for the white-noise case.

We will consider a general process defined by the Langevin equation

$$
\dot{q}=v(q)+g(q) p(t),
$$

where $v$ and $g$ are general nonlinear functions of $q$, and $p(t)$ is an Ornstein-Uhlenbeck noise whose correlation is given by Eq. (2.2).

The non-Markovian correlation function $C(t)$ is defined by

$$
C(t)=\lim _{t^{\prime} \rightarrow \infty} \frac{\left\langle\delta q\left(t+t^{\prime}\right) \delta q\left(t^{\prime}\right)\right\rangle}{\left\langle(\delta q)^{2}\right\rangle},
$$


where $\delta q(t)=q(t)-\langle q\rangle$ and $\left\langle q^{n}\right\rangle$ are the steady-state moments. To first order in $\tau, C(t)$ can be expressed as [17]

$$
C(t)=C_{M}(t)+\tau\left[1-\exp \left[-\frac{t}{\tau}\right]\right] \gamma_{0} C_{M}^{L}(t)+o\left(\tau^{2}\right),
$$

where $\gamma_{0}=-\left(\left\langle\delta q L^{+} \delta q\right\rangle\right) /\left[\left\langle(\delta q)^{2}\right\rangle\right]$ and

$$
C_{M}(t)=\frac{\langle\delta q(t) \delta q\rangle_{M}}{\left\langle(\delta q)^{2}\right\rangle}
$$

and

$$
C_{M}^{L}(t)=\frac{\left\langle\delta q(t) L^{+}(\tau) \delta q\right\rangle_{M}}{\left\langle\delta q L^{+}(\tau) \delta q\right\rangle}
$$

are correlation functions associated with an effective Markovian process defined by a Fokker-Planck operator $L(\tau)$ given by Eqs. (2.4) and (2.5).

Two remarks about the general features of Eq. (3.3) are in order. First, it contains the characteristic initial plateau, associated with the vanishing first derivative at $t=0$, of any non-Markovian correlation function $[5,26,27]$. Second, for $t \gg \tau$, it corresponds to the decay of the correlation function of a Markovian process with an effective initial condition.

The next step in our procedure consists of the application of the double-expansion method $[18,19]$ to $C_{M}(t)$ and $C_{M}^{L}(t)$. The basic idea is to consider, for any Markovian correlation function, two expansions of the Laplace transform, both for high and low frequencies $\omega$ :

$$
C_{M}(\omega)=\left\{\begin{array}{l}
\frac{1}{\omega} \sum_{k=0}^{\infty} \mu_{M}^{k}\left(\frac{1}{\omega}\right)^{k} \\
\sum_{k=0}^{\infty} \frac{(-1)^{k}}{k !} T_{M}^{k} \omega^{k},
\end{array}\right.
$$

where the coefficients of the expansions are related to the derivatives at $t=0, \mu_{M}^{k}$, and the relaxation moments $T_{M}^{k}$ as

$$
\begin{aligned}
\mu_{M}^{k} & =\left.\frac{d^{k}}{d t^{k}} C_{M}(t)\right|_{t=0}, \\
T_{M}^{k} & =\int_{0}^{\infty} C_{M}(t) t^{k} d t .
\end{aligned}
$$

Explicit expressions for these quantities are given in Refs. [17] and [19]. These coefficients contain information on the small and large time scales, respectively. The goal of the method is to combine simultaneously information from both expansions by means of Padé interpolation in the $\omega$ space. As discussed in Refs. [17] and [19], an approximation for $C_{M}(t)$ containing in an exact way $n$ derivatives and $m$ relaxation moments (" $n d m t$ " approximation) is given by a superposition of $N$ exponentials of the form

$$
C_{M}^{N}(t)=\sum_{n=1}^{N} a_{n} e^{-\lambda_{n} t}
$$

where $n+m+1=2 N, C_{M}^{N}(0)=1$, and

$$
\begin{aligned}
& \sum_{i=1}^{N} a_{i}\left(\lambda_{i}\right)^{k}=(-1)^{k} \mu_{M}^{k} \quad(k=0,1, \ldots, n), \\
& \sum_{i=1}^{N} a_{i}\left(\lambda_{i}\right)^{-k-1}=\frac{T_{M}^{k}}{k !} \quad(k=0,1, \ldots, m-1) .
\end{aligned}
$$

\section{B. Application and comparison with simulations of the gain-noise model}

Here we discuss some different orders of approximation of the method for $C(t)$. From Eq. (3.3) one can argue that $C_{M}^{L}(t)$ only contributes essentially for long times, whereas $C_{M}(t)$ is important both in the short- and longtime regimes [17]. Therefore, at the lowest order, imposing the condition for the first derivative on $C_{M}(t)$ and the zeroth-order relaxation moment (the relaxation time) on $C_{M}^{L}(t)$ we get

$$
C(t)=e^{-\gamma_{0} t}+\tau \gamma_{0}\left(1-e^{(-t / \tau)}\right) e^{-\gamma_{0} t} .
$$

This approximation contains the exact first derivative of the non-Markovian $C(t)$. To get the right relaxation time of $C(t)$ to order $\tau$ and the same exact derivative, the next simplest approximation is

$$
\begin{aligned}
C(t)= & {\left[a_{1} e^{-\lambda_{1} t}+\left(1-a_{1}\right) e^{-\lambda_{2} t}\right] } \\
& +\tau \gamma_{0}\left[1-\exp \left[-\frac{t}{\tau}\right]\right] e^{-\gamma_{0} t},
\end{aligned}
$$

where $a_{1}, \lambda_{1}$, and $\lambda_{2}$ are determined by imposing one derivative and two relaxation moments of $C_{M}(t)$.

In order to apply the method to the gain-noise model we will consider an effective Markovian Fokker-Planck operator $L$, in which the diffusion coefficient is given by Eq. (2.7). In this way the spurious boundaries are avoided.

In Fig. 1 we compare the approximations (3.13) and (3.14) for the gain-noise model with $\alpha / \alpha_{1}=0.2,\langle I\rangle=1$, and $\tau=0.25$. We plot the correlation function $\lambda(t)$ given

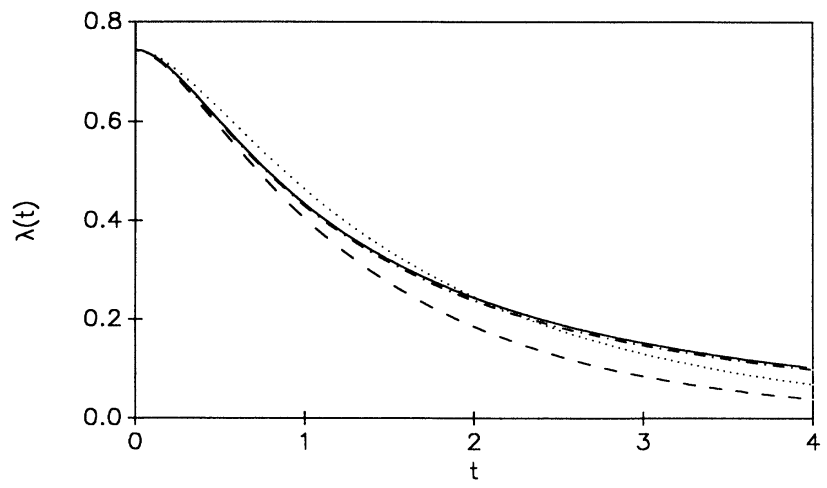

FIG. 1. Intensity correlation function $\lambda(t)$ for the coloredgain-noise model with $\alpha / \alpha_{1}=0.2,\langle I\rangle=1$, and $\tau=0.25$. The value of $\tau / t_{d}$ is 0.16 . - simulation; - - $-1 d 0 t$ approximation; -. -.-., $1 d 2 t$ approximation; . . . . linearization. 


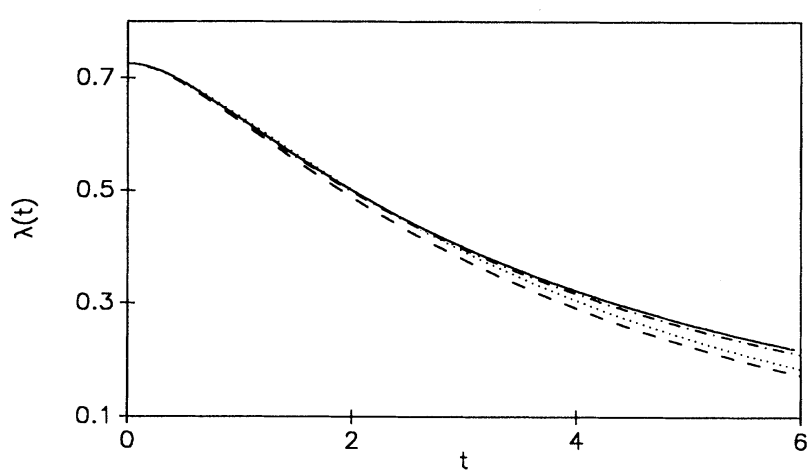

FIG. 2. Intensity correlation function $\lambda(t)$ for the coloredgain-noise model with $\alpha / \alpha_{1}=0.5,\langle I\rangle=1$, and $\tau=0.5$. The value of $\tau / t_{d}$ is 0.125 . - , simulation; - -, $1 d 0 t$ approximation; - - - - - , $1 d 2 t$ approximation; . . . linearization.

by (2.14), i.e., $\lambda(t)=C(t)\left(\left\langle I^{2}\right\rangle-\langle I\rangle^{2}\right) /\langle I\rangle^{2}$. We see an excellent agreement between the simulation [28] and approximation (3.14) for the complete-time regime. Even for this relatively low order of approximation, the method contains the nonlinear effects accurately, as we see by comparison with the linear approximation (2.15). On the other hand, the approximation (3.13) also contains the correct initial plateau but only describes correctly the decay at short times.

In Fig. 2 we take $\langle I\rangle=1, \tau=0.5$, and $\alpha / \alpha_{1}=0.5$, so we are going away from the loss-noise model. We again find a good agreement between simulation and approximation (3.14). The linear approximation in Figs. 1 and 2 suggests that the nonlinear effects are reduced when $\alpha / \alpha_{1}$ increases.

For the range of parameters we are considering in this paper, above threshold, a criterion for the range of validity of the small- $\tau$ approximation introduced in Eq. (3.3) is given by the ratio $\tau / t_{d} \ll 1$, where $t_{d}$ is the deterministic

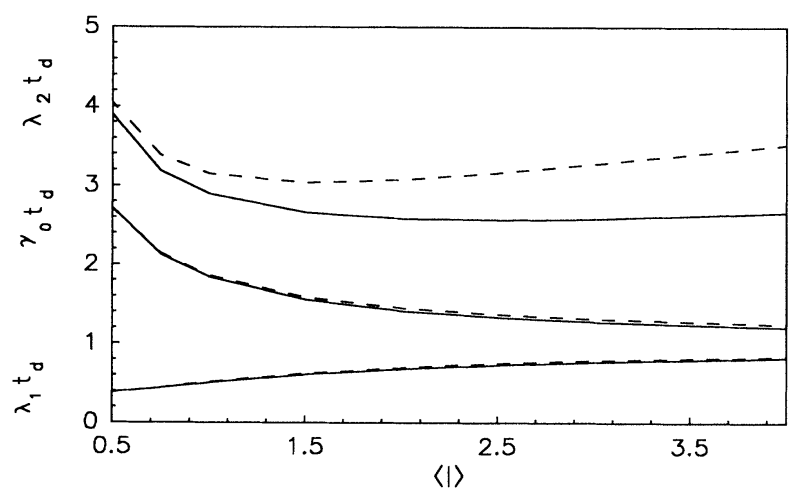

FIG. 3. Time scales corresponding to the three exponentials used to describe the normalized intensity correlation function $C(t)$, rescaled with the deterministic time $t_{d}$, vs the mean intensity $\langle I\rangle$, for the loss-noise model, $\alpha / \alpha_{1}=0$,: $\gamma_{0} t_{d}$ (both curves in the middle), time scale corresponding to $C_{M}^{L}(t) ; \lambda_{1} t_{d}$ (both curves on the bottom), dominant time scale of $C_{M}(t)$; and $\lambda_{2} t_{d}$ (curves on the top), dominated time scale of $C_{M}(t)$. In the three cases the values of $\tau$ are,$- 0.1 ;---, 0.25$.

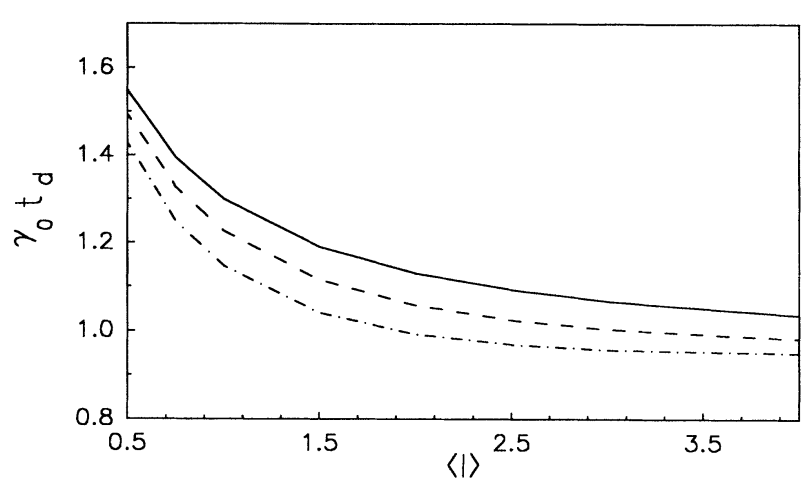

FIG. 4. Time scale $\gamma_{0}$ rescaled with $t_{d}, \gamma_{0} t_{d}$, vs the mean intensity $\langle I\rangle$, for $\alpha / \alpha_{1}=0.2$ and different values of $\tau$ :,- 0.1 ; ,$--- 0.25 ;-\cdot-\cdot-\cdot, 0.5$.

relaxation time given by the linear approximation [see Eq. (2.13)]. For Figs. 1 and $2, \tau / t_{d}$ is 0.16 and 0.125 , respectively. We have checked that the approximation (3.14) is valid when $\tau / t_{d} \leq 0.3$. This criterion implies that an increase of $\langle I\rangle$ reduces the range of validity of the small- $\tau$ approximation (3.3), due to the fact that it also implies a decrease of $t_{d}$ [see Eq. (2.13)], despite the fact that the nonlinear effects are reduced in this case [see Eq. (2.16)].

We have also checked that the approximation (3.14) gives good results for small $\tau$ for the colored-loss-noise model.

\section{DECAY OF THE INTENSITY CORRELATION FUNCTIONS}

First, we analyze the limit $\tau \gg t_{d}$. In that case, we assume $\partial_{t} I=0$ in Eqs. (2.1) and (2.3) (adiabatic approximation [16]). With this approximation, we get

$$
I(t)=\alpha /\left(1-\alpha / \alpha_{1}\right)+\left(1-\alpha / \alpha_{1}\right)^{-1} p(t)
$$

for the gain-noise model. Then, the normalized correlation function $C(t)$ [see Eq. (3.2)] is the same for both models, and it coincides with the one corresponding to the noise. This result agrees with experimental observations [8] and numerical simulations [11].

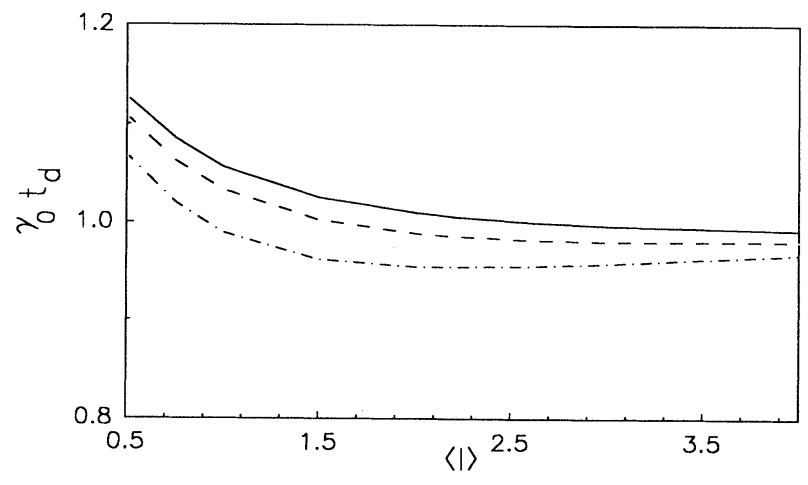

FIG. 5. Time scale $\gamma_{0}$ rescaled with $t_{d}, \gamma_{0} t_{d}$, vs the mean intensity $\langle I\rangle$, for $\alpha / \alpha_{1}=0.5$ and different values of $\tau$ : $0.25 ;---, 0.5 ;-\cdot-\cdot-\cdot, 1$. 


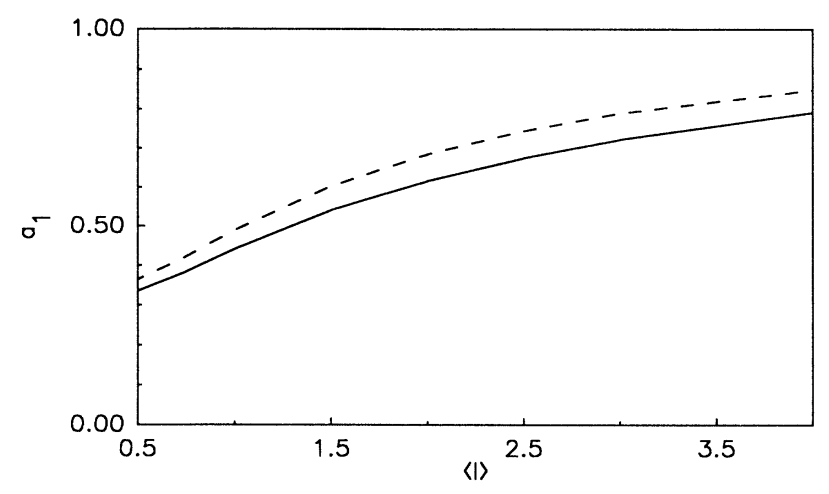

FIG. 6. The amplitude $a_{1}$ of the first exponential used to approximate the effective Markovian correlation function $C_{M}(t)$ vs the mean intensity $\langle I\rangle$ for $\alpha / \alpha_{1}=0$ (loss-noise model). The amplitude of the second exponential is $\left(1-a_{1}\right)$. Same parameters as in Fig. 3.

Now, we analyze the decay of $C(t)$ for correlation times smaller than the deterministic time $\tau<t_{d}$. For that, we use Eq. (3.14) from the method presented in Sec. III. We have shown in Sec. III that the method gives excellent results when $\tau<<t_{d}$. In the following, we analyze the behavior of the time scales involved in the decay of $C(t)$ with increasing $\langle I\rangle$ for different values of $\alpha / \alpha_{1}$ and $\tau$. Due to the decrease of $t_{d}$ with $\langle I\rangle$ [see (2.13)], it is clear that the validity of the method is reduced when $\langle I\rangle$ increases. In our analysis of the decay of $C(t)$ we will focus on qualitative results. In this sense, our conclusion remains valid even when $\tau$ approaches $t_{d}$.

We consider three values of $\alpha / \alpha_{1}: 0$ (loss-noise model), 0.2 , and 0.5 . Regarding the correlation time, it can be seen from Eq. (2.13) that $t_{d}$ increases when $\alpha / \alpha_{1}$ does. Then, larger correlation times are required to have nonnegligible non-Markovian effects when $\alpha / \alpha_{1}$ is increased. We have considered the following values of $\tau: 0.1$ and 0.25 for $\alpha / \alpha_{1}=0, \tau=0.1,0.25$, and 0.5 for $\alpha / \alpha_{1}=0.2$, and $\tau=0.25,0.5$, and 1 for $\alpha / \alpha_{1}=0.5$.

For times shorter than $\tau$, the only aspect that plays an important role is the initial rounding off of the intensity

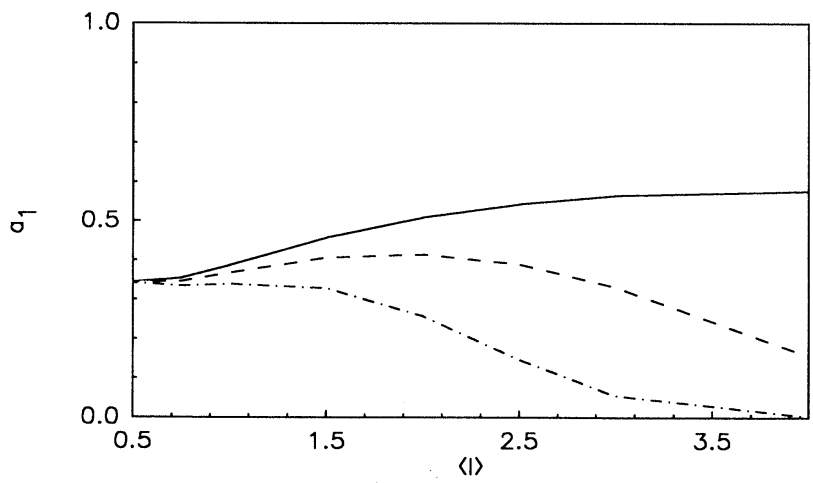

FIG. 7. The amplitude $a_{1}$ of the first exponential used to approximate the effective Markovian correlation function $C_{M}(t)$ vs the mean intensity $\langle I\rangle$ for $\alpha / \alpha_{1}=0.2$. Same parameters as in Fig. 4.

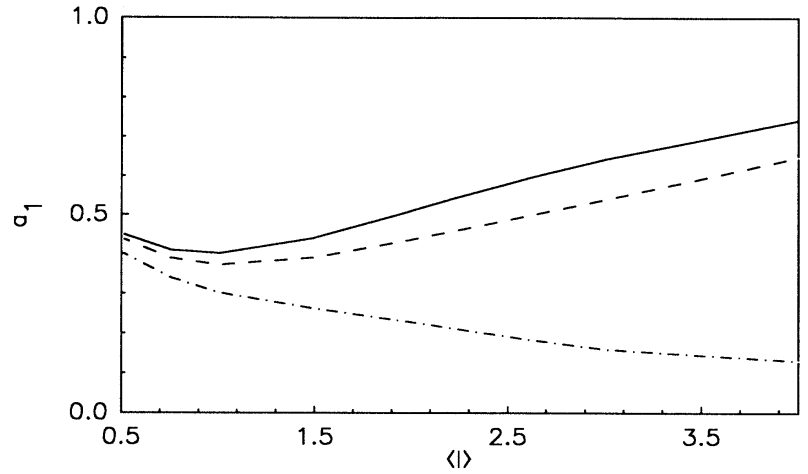

FIG. 8. The amplitude $a_{1}$ of the first exponential used to approximate the effective Markovian correlation function $C_{M}(t)$ vs the mean intensity $\langle I\rangle$ for $\alpha / \alpha_{1}=0.5$. Same parameters as in Fig. 5.

correlation functions. Then, in order to analyze the decay of $C(t)$ we consider times $t \gg \tau$. In Eq. (3.14) three time scales appear, and we first consider the last term. For $t \gg \tau$, the amplitude of the third term is $\gamma_{0} \tau$. If this amplitude is very small, it can be neglected, and the behavior of $C(t)$ coincides with that of $C_{M}(t)$, but when $\tau$ increases it is necessary to take $C_{M}^{L}(t)$ into account. We represent in Figs. 3, 4, and $5 \gamma_{0}$ rescaled with $t_{d}$. In the loss-noise model (Fig. 3), $\gamma_{0}^{-1}$ approaches $t_{d}$ when the mean intensity increases. For the gain-noise model, it is observed from Figs. 4 for $\alpha / \alpha=0.2$ and 5 for $\alpha / \alpha_{1}=0.5$ that $\gamma_{0}^{-1}$ approaches $t_{d}$ when $\tau$ and $\langle I\rangle$ increase.

In the following we analyze the decay of $C_{M}(t)$, which is given by the time scales $\lambda_{1}, \lambda_{2}$, and their amplitudes $a_{1}$ and $\left(1-a_{1}\right)$, respectively. We compare both models as $\tau$ increases. We show in Figs. 6, 7, and $8 a_{1}$ for $\alpha / \alpha=0$, 0.2 , and 0.5 , respectively. For the loss-noise model, $a_{1}$ grows with $\langle I\rangle$ and $\tau$. This means that the dominant time scale tends to be $\lambda_{1}$. In the other two figures, for the gain-noise model, we see, in contrast to the white-noise case [12] and to the loss-noise model, that when $\tau$ and $\langle I\rangle$ increase, $a_{1}$ decreases, so for $\tau$ or $\langle I\rangle$ large enough

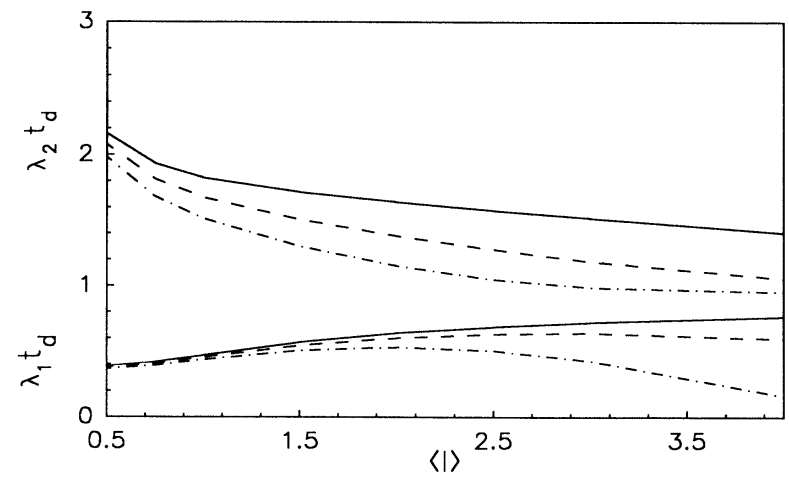

FIG. 9. Time scales corresponding to both exponentials used to approximate $C_{M}(t)$, rescaled with $t_{d}$, vs the mean intensity $\langle I\rangle$ for $\alpha / \alpha_{1}=0.2: \lambda_{2} t_{d}$, dominant time scale (three curves on the top), and $\lambda_{1} t_{d}$, dominated time scale (three curves on the bottom). In both cases, the parameters are as in Fig. 4 . 


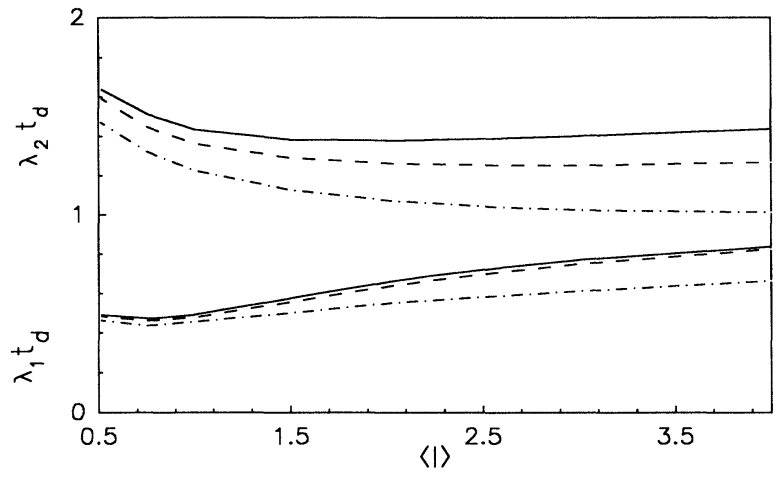

FIG. 10. Time scales corresponding to both exponentials used to approximate $C_{M}(t)$, rescaled with $t_{d}$, vs the mean intensity $\langle I\rangle$ for $\alpha / \alpha_{1}=0.5: \lambda_{2} t_{d}$, dominant time scale (three curves on the top), and $\lambda_{1} t_{d}$, dominated time scale (three curves on the bottom). In both cases, the parameters are as in Fig. 5

the dominant time scale is $\lambda_{2}$.

In Figs. 3, 9, and 10 we represent the dominant time scales of both models, that is, $\lambda_{1}$ for the loss-noise model and $\lambda_{2}$ for the gain-noise model, in all cases rescaled with $t_{d}$. In Fig. 3 we see that $\lambda_{1}^{-1}$ approaches $t_{d}$ when $\tau$ increases. The same situation is observed in Figs. 9 and 10 for the dominant time scale $\lambda_{2}$ of the gain-noise model. Therefore, in all cases, the dominant time scales approach the deterministic time $t_{d}$ and, in this sense, the differences between the gain- and the loss-noise model tend to disappear with $\tau$, in agreement with simulation results [15]. As we have shown above, the third time scale $\gamma_{0}$ behaves in a similar way. Then, it does not modify essentially the decay of $C(t)$ when the mean intensity and $\tau$ increase.
Now, we analyze the dominated time scale, that is, $\lambda_{1}$ in the gain-noise model and $\lambda_{2}$ in the loss-noise model. We represent these scales in Figs. 3, 9, and 10, again rescaled with $t_{d}$. We see in Fig. 3 that $\lambda_{2} t_{d}$ is always larger than 1 , and increases when $\tau$ does. So, $\lambda_{2}^{-1}$ is always smaller than $t_{d}$, and it can be seen that it is also smaller than $\tau$. Then, we can consider that the main contribution to the decay of $C(t)$ comes from the time scales $\lambda_{1}$ and $\gamma_{0}$, which approach $t_{d}$ when the mean intensity and $\tau$ increase. On the contrary, in Figs. 9 and 10 we observe another tendency: $\lambda_{1} t_{d}$ is always smaller than 1 for $\alpha / \alpha_{1}=0.2$ and 0.5 . The conclusion is that as $\tau$ increases, a difference remains between both models, and that is given by the dominated time scale. This time scale is larger than $t_{d}$ in the gain-noise model, so the decay of $C(t)$ is slower in this case than in the loss-noise model, in which the dominated scale is smaller than $t_{d}$.

Our findings concerning the decay of the intensity correlation function can be summarized as follows. When $\tau \gg t_{d}$ (far above threshold), the correlation functions of both models are determined by the pump noise. For correlation times smaller than $t_{d}$, the decay of $C(t)$ for both models can be described with increasing $\tau$ and $\langle I\rangle$ by a time scale that approaches the deterministic time. However, a larger time scale remains for the gainnoise model, which could allow us to distinguish between both models.

\section{ACKNOWLEDGMENTS}

Financial support from Dirección General de Investigación Científica y Técnica (Spain), Project PB87-0014, is acknowledged. Two of us (J.C. and D.H-M.) acknowledge financial support of the European Economic Community through Project No. SC1.0043.C(H).
[1] J. A. Abate, H. J. Kimble, and L. Mandel, Phys. Rev. A 14, 788 (1976).

[2] K. Kaminishi, R. Roy, S. Short, and L. Mandel, Phys. Rev. A 24, 370 (1981); R. Short, L. Mandel, and R. Roy, Phys. Rev. Lett. 49, 647 (1982).

[3] R. Graham, H. Hohnerbach, and A. Schenzle, Phys. Rev. Lett. 48, 1396 (1982); P. Jung, Th. Leiber, and H. Risken, Z. Phys. B 66, 397 (1987).

[4] S. N. Dixit, and P. S. Shani, Phys. Rev. Lett. 50, 1273 (1983); A. Schenzle and R. Graham, Phys. Lett. A 98, 319 (1983); P. Jung, and H. Risken, ibid. 103, 38 (1984); P. Jung, Th. Leiber, and H. Risken, Z. Phys. B 68, 123 (1987).

[5] A. Hernández-Machado, M. San Miguel, and S. L. Katz, Phys. Rev. A 31, 2362 (1985).

[6] R. Roy, A. W. Yu, and S. Zhu, in Noise in Nonlinear Dynamical Systems, edited by F. Moss and P. V. E. McClintock (Cambridge University Press, Cambridge, England, 1989), Vol. III, p. 90.

[7] M. San Miguel, in Instabilities and Chaos in Quantum Optics II, edited by N. B. Abraham, F. T. Arecchi, and L. A. Lugiato (Plenum, New York, 1988), p. 147.

[8] A. W. Yu, G. P. Agrawal, and R. Roy, Opt. Lett. 12, 806
(1987).

[9] P. D. Lett and E. C. Gage, Phys. Rev. A 39, 1193 (1989).

[10] M. Aguado, E. Hernández-García, M. San Miguel, Phys. Rev. A 38, 5670 (1988).

[11] E. Hernández-García, M. San Miguel, R. Toral, and M. Aguado, in Coherence and Quantum Optics VI, edited by L. Mandel and E. Wolf (Plenum, New York, 1989), p. 483.

[12] J. M. Noriega, L. Pesquera, and M. A. Rodríguez, Phys. Rev. A 43, 4008 (1991).

[13] A. Schenzle, J. Stat. Phys. 54, 1243 (1989).

[14] E. Peacock-López, F. Javier de la Rubia, B. J. West, and K. Lindenberg, Phys. Rev. A 39, 4026 (1989).

[15] E. Hernández-García, R. Toral, and M. San Miguel, Phys. Rev. A 42, 6823 (1990).

[16] R. L. Stratonovich, Topics in the Theory of Random Noise (Gordon and Breach, New York, 1967), Vol. II.

[17] A. Hernández-Machado, J. Casademunt, M. A. Rodríguez, L. Pesquera, and J. M. Noriega, Phys. Rev. A 43, 1744 (1991).

[18] W. Nadler and K. Schulten, J. Chem. Phys. 82, 151 (1985); Z. Phys. B 59, 53 (1985).

[19] J. Casademunt and A. Hernández-Machado, Z. Phys. B 76, 403 (1989). 
[20] A. Hernández-Machado, M. San Miguel, and J. M. Sancho, Phys. Rev. A 29, 3388 (1984).

[21] J. M. Sancho and M. San Miguel, in Fluctuations and Sensitivity in Nonequilibrium Systems, edited by $\mathrm{W}$. Horsthemke and D. K. Kondepudi (Springer-Verlag, Berlin, 1984); J. Casademunt, R. Mannella, P. V. E. McClintock, F. Moss, and J. M. Sancho, Phys. Rev. A 35, 5183 (1987).

[22] J. M. Sancho, M. San Miguel, S. Katz, and J. D. Gunton, Phys. Rev. A 26, 1589 (1982).

[23] M. Aguado and M. San Miguel, Phys. Rev. A 37, 450 (1988).

[24] P. Jung and P. Hanggi, Phys. Rev. A 35, 4464 (1987).

[25] R. Fox, Phys. Rev. A 34, 4525 (1986).
[26] M. San Miguel, L. Pesquera, M. A. Rodríguez, and A. Hernández-Machado, Phys. Rev. A 35, 208 (1987).

[27] L. Pesquera and M. A. Rodríguez, in Recent Developments in Nonequilibrium Thermodynamics: Fluids and Related Topics, edited by J. Casas-Vázquez and D. Jou, Lecture Notes in Physics Vol. 253 (Springer-Verlag, Berlin, 1986).

[28] The numerical simulations have been done following the algorithm explained in Ref. [22]. The correlation function is obtained averaging over 3000 trajectories and over 30000 integration steps in each trajectory. To assure that the stationary state has been reached, we eliminate the first 7500 integration steps. We used an integration step $\Delta=0.005$. 